\title{
Kernos
}

Revue internationale et pluridisciplinaire de religion grecque antique

$18 \mid 2005$

Varia

\section{Héraclès, Tyndare et Hippocoon dans la description de Sparte par Pausanias}

Mise en espace d'une tradition mythique

\section{Olivier Gengler}

\section{(2) OpenEdition \\ Journals}

Édition électronique

URL : http://journals.openedition.org/kernos/1545

DOI : 10.4000/kernos. 1545

ISSN : 2034-7871

Éditeur

Centre international d'étude de la religion grecque antique

\section{Édition imprimée}

Date de publication : 1 janvier 2005

Pagination : 311-328

ISSN : 0776-3824

\section{Référence électronique}

Olivier Gengler, « Héraclès, Tyndare et Hippocoon dans la description de Sparte par Pausanias », Kernos [En ligne], 18 | 2005, mis en ligne le 08 juillet 2011, consulté le 02 mai 2019. URL : http:// journals.openedition.org/kernos/1545; DOI : 10.4000/kernos. 1545 


\title{
Héraclès, Tyndare et Hippocoon dans la description de Sparte par Pausanias : mise en espace d'une tradition mythique
}

\begin{abstract}
Résumé : Qu'il soit placé à l'origine des revendications des Héraclides ou instrumentalisé dans le cadre de l'opposition séculaire entre Messéniens et Spartiates, l'épisode du combat d'Héraclès contre Hippocoon et ses fils occupe une place de choix dans le passé de Sparte tel que le transmet Pausanias. Aussi vénérables qu'ils paraissent, les éléments de cette tradition et les monuments spartiates qui lui sont liés s'intègrent néanmoins très concrètement dans l'horizon politique et religieux du II $^{\mathrm{e}}$ siècle ap. J.-C. Le discours développé dans la Périégèse semble d'ailleurs bien répondre à des préoccupations similaires à celles qui animent les grandes familles spartiates de cette époque.
\end{abstract}

Abstract: Heracles, Tyndareus, and Hippocoon in Pausanias' Description of Sparta: mapping out a mythical tradition. Placed at the origin of the claims of the Heraclids or instrumented within the framework of the secular conflict between Messenians and Spartans, the episode of the fight of Heracles against Hippocoon and its sons occupies an important place in the Spartan past, just as Pausanias states. As ancient as they appear, the elements of this tradition and the Spartan monuments related to it integrate nevertheless very concretely into the political and religious context of the IInd c. AD. The purpose of the Periegesis seems moreover to meet needs quite similar to those preoccupying the great Spartan families at this time.

En plusieurs endroits de sa Périégèse, Pausanias fait allusion à l'opposition entre Tyndare et Hippocoon et au combat qui opposa ce dernier, ainsi que ses fils, à Héraclès. Dans la généalogie des rois d'Argos tout d'abord, il évoque ces événements à propos des prétentions des Héraclides sur chacune des trois grandes régions doriennes historiques du Péloponnèse. C'est précisément à la victoire d'Héraclès sur Hippocoon que les Héraclides devaient de pouvoir revendiquer le trône de Sparte, conquis par Héraclès et laissé à Tyndare pour en assurer la régence ${ }^{1}$ :

Argos et la royauté à Argos, ils la revendiquaient le plus légitimement, à ce qu'il me semble, puisque Tisamène était un Pélopide, tandis que les Héraclides étaient dès l'origine des Perséides. Ils firent valoir que Tyndare lui-même avait été destitué par Hippocoon, mais Héraclès, affirmèrent-ils, après avoir tué Hippocoon et ses enfants, confia la région en dépôt à Tyndare. Tel est aussi ce qu'ils disaient à propos de la Messénie, à savoir qu'elle avait été donnée en dépôt à Nestor par Héraclès après qu'il eut pris Pylos. De Lacédémone et d'Argos, ils expulsèrent donc Tisamène et de Messénie les descendants de Nestor.

\footnotetext{
${ }^{1}$ Pausanias, II, 18, 7. Nos traductions suivent le texte établi par M.H. ROCHA-PEREIRA, Pausaniae Graeciae Descriptio, t. II, Leipzig, 19912 [1977]. Sur l'ensemble de ce passage, voir M. PIÉRART, "Pausanias et les généalogies d'Argos : étude de quelques problèmes », in D. Auger \& S. SAÏD, Généalogies mythiques, Paris, 1998, p. 141-161.
} 
Une seconde allusion apparaît dans la généalogie des rois de Sparte. Pausanias ne revient plus sur les revendications des Héraclides et se concentre plutôt sur les implications que la lutte de succession a eues pour Tyndare $^{2}$ :

Ce dernier [Oibalos, un descendant de Lacédémon] eut pour femme Gorgophonè, la fille de Persée, originaire d'Argos et eut pour enfant Tyndare, à qui Hippocoon ne cessa de disputer la royauté estimant mériter le pouvoir en vertu du droit d'aînesse. Allié à Icarios et aux rebelles il surpassa largement Tyndare par sa puissance et contraignit celui-ci à s'exiler, effrayé, à Pellana, comme l'affirment les Lacédémoniens; mais à ce sujet les Messéniens tiennent un discours selon lequel Tyndare, en fuite, est venu chez Apharée en Messénie et selon lequel Apharée, fils de Périérès, était par sa mère le frère de Tyndare; et ce dernier a vécu à Thalamai de Messénie, affirment-ils, et ses enfants sont nés alors qu'il vivait là-bas. Quelque temps plus tard, Tyndare revint grâce à Héraclès et il recouvra le pouvoir.

La trame narrative de l'épisode rapporté à propos des Héraclides - à savoir : Hippocoon chasse Tyndare, Héraclès tue Hippocoon et ses enfants, Héraclès remet Tyndare sur le trône - se retrouve en partie ici, mais focalisée sur Tyndare: Hippocoon chasse Tyndare, Tyndare séjourne hors de Sparte, Tyndare retrouve son trône. Par ce changement de perspective, Pausanias associe chaque présentation des mêmes événements à des débats aux enjeux historiques et territoriaux différents : le retour des Héraclides dans le Péloponnèse d'une part, la dissension entre Lacédémoniens et Messéniens de l'autre. Ces deux perspectives se prolongent et se mêlent dans la suite du texte de Pausanias où, progressivement, le récit complet des événements va se construire. Nous allons en suivre le développement dans les Lakônika et voir dans quelle mesure le discours de Pausanias trouve un écho dans la cité de Sparte de la fin du II ${ }^{\mathrm{e}}$ siècle ap. J.-C. ${ }^{3}$.

\section{Héraclès, Hippocoon et les Hippocoontides}

Plusieurs monuments directement liés à la lutte d'Héraclès contre Hippocoon et ses fils jalonnent la cité de Sparte et ses environs tels que nous les décrit Pausanias. Le premier se trouve sur la route qui va d'Hermai - «les

\footnotetext{
2 Paus., III, 1, 4-5. Pour une analyse du début des Lakônika de Pausanias, voir C. CALAME, "Le récit généalogique spartiate : la représentation mythologique d'une organisation spatiale ", QS 26 (1987), p. 43-91, spécialement p. 62-65.

3 Nous nous attacherons donc essentiellement ici à la place que Pausanias attribue à la lutte d'Héraclès contre Hippocoon et ses fils dans sa Périégèse. Pour l'interprétation de cet épisode évoqué dans le Parthénée d'Alcman (fr. 3 Calame) - dans le cadre des rituels d'adolescence spartiate à l'époque archaïque, voir C. CALAME, Les Chours de jeunes filles en Grèce archaïque, t. II : Alcman, Rome, 1977 (Filologia e critica, 21), p. 52-58, avec O. GENGLER, «Les Dioscures et les Apharétides dans le Parthénée d'Alcman », LEC 63 (1995), p. 1-21. Pour une lecture centrée sur la figure civilisatrice d'Héraclés, voir M. Giangiulio, "Le héros fondateur, l'espace sacré de la déesse. Notes sur Héraclès et les sanctuaires d'Héra du Péloponnèse à la Grande Grèce », in C. Jourdain-ANnequin \& C. BOnNet (éds), II Rencontre héracléenne: Héraclès et le féminin, Bruxelles/Rome, 1996 (Inst. Hist. belge de Rome, Études de philologie, d'archéologie et d'bistoire ancienne, 31), p. 215-233.
} 
Hermès » - à Sparte. "Les Hermès » se trouvaient dans le Parnon au point de rencontre des «frontières des Lacédémoniens avec les Argiens et les Tégéates ${ }^{4}$. Sur la gauche de cette route, après le lieu-dit Skotitas et avant Karyai, se trouvait « une statue d'Héraclès et un trophée : Héraclès, disait-on l'avait érigé après avoir tué Hippocoon et ses enfants $»^{5}$. La mention de ce monument apparaît, dans le texte de Pausanias, après les deux passages que nous avons déjà lus, mais, pour sa teneur, il renvoie seulement au premier. Dans la généalogie des rois de Sparte en effet, lorsqu'il rapporte les déboires de Tyndare, Pausanias ne mentionne pas la mort d'Hippocoon, et encore moins celle de ses enfants qui n'étaient nullement impliqués dans la querelle dynastique telle qu'il l'a rapportée.

Ce sont néanmoins les fils d'Hippocoon qui vont reparaître ensuite, dans la description de Sparte. Pausanias signale en effet les hérôa de pas moins de six d'entre eux en rappelant chaque fois leur filiation ${ }^{6}$. Le monument funéraire d'Eumédès est le premier monument que signale Pausanias à proximité du Dromos, la «Piste de course » où, du temps du Périégète encore, les jeunes gens ( véol) s'entraînaient à la course $e^{7}$. Tout de suite après la mention du monument funéraire d'Eumédès, Pausanias signale une statue ancienne d'Héraclès à laquelle sacrifient les sphaireis, c'est-à-dire les jeunes hommes quittant l'éphébie.

Un peu plus loin dans la description apparaît la mention de l'bérôon d'Alcon situé non loin du début du Dromos où se trouvaient les Dioscures Aphétèrioi ${ }^{8}$.

\footnotetext{
${ }^{4}$ Paus., II, 38, 7. Voir la carte ci-dessous. "Les Hermès » devaient se trouver au lieu-dit Phénomenoi : P.B. Phaklaris, Archaia Kynouria, Athènes, 1990, p. 193-195 (avec la bibliographie); le site a été fouillé : K. RHomaios, «Laconia, IV: The 'Equxi on the N.E. Frontier », ABSA

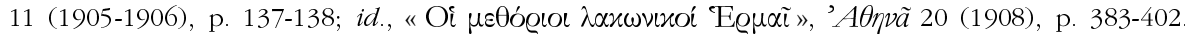
Aperçu des trouvailles chez D.G.J. SHIPLEY, «Archaeological sites in Laconia », in W.G. CAVANAGH, J.H. Crouwel, R.W.V. Catuing \& D.G.J. ShiPley (éds), Continuity and Change in a Greek Rural Landscape: the Laconia Survey, II: Archaeological Data, Londres, 1996 (BSA, Supplementary vol. 27), p. 280, site AA24. L'évolution du territoire de la cité de Sparte a été étudiée par D.G.J. SHIPLEY, "The extent of Spartan territory in the Late Classical and Hellenistic periods », ABSA 95 (2000), p. 367-390 que l'on complètera par P. CARTLEDGE \& A. SPAWFORTH, Hellenistic and Roman Sparta. A Tale of two Cities, Londres, 2002 [1989] (States and Cities of Ancient Greece), p. 136-142 pour l'époque romaine.

5 Paus., III, 10, 6. L'identification des sites de Skotitas et de Karyai est sujette à discussion. Résumé dans SHIPLEY, "Archaeological sites in Laconia », l.c. (n. 4), p. 284-285, sites DD45, DD46 et DD47.

${ }^{6}$ Apollodore, III, 10, 5 (124) nomme douze fils d'Hippocoon, parmi lesquels se trouvent cinq des six personnages dont Pausanias signale les monuments à Sparte; seul Eumédès manque à l'appel. D'après Diodore, IV, 33, les fils d'Hippocoon étaient vingt, dont dix périrent dans le combat contre Héraclès, soit la moitié d'entre eux.

Paus., III, 14, 6. Sur le Dromos spartiate, voir P. MARCHETTI, "Le 'Dromos' au cœur de l'agora de Sparte : les dieux protecteurs de l'éducation en pays dorien. Points de vue nouveaux », Kernos 9 (1996), p. 155-170. L'éducation des jeunes gens à Sparte à l'époque romaine a fait l'objet d'une étude d'ensemble par N. Kennelt, The Gymnasium of Virtue: Education and Culture in ancient Sparta, Chapel Hill/Londres, 1995, part. chapitres 1 à 4 .

${ }^{8}$ Paus., III, 14, 7.
} 


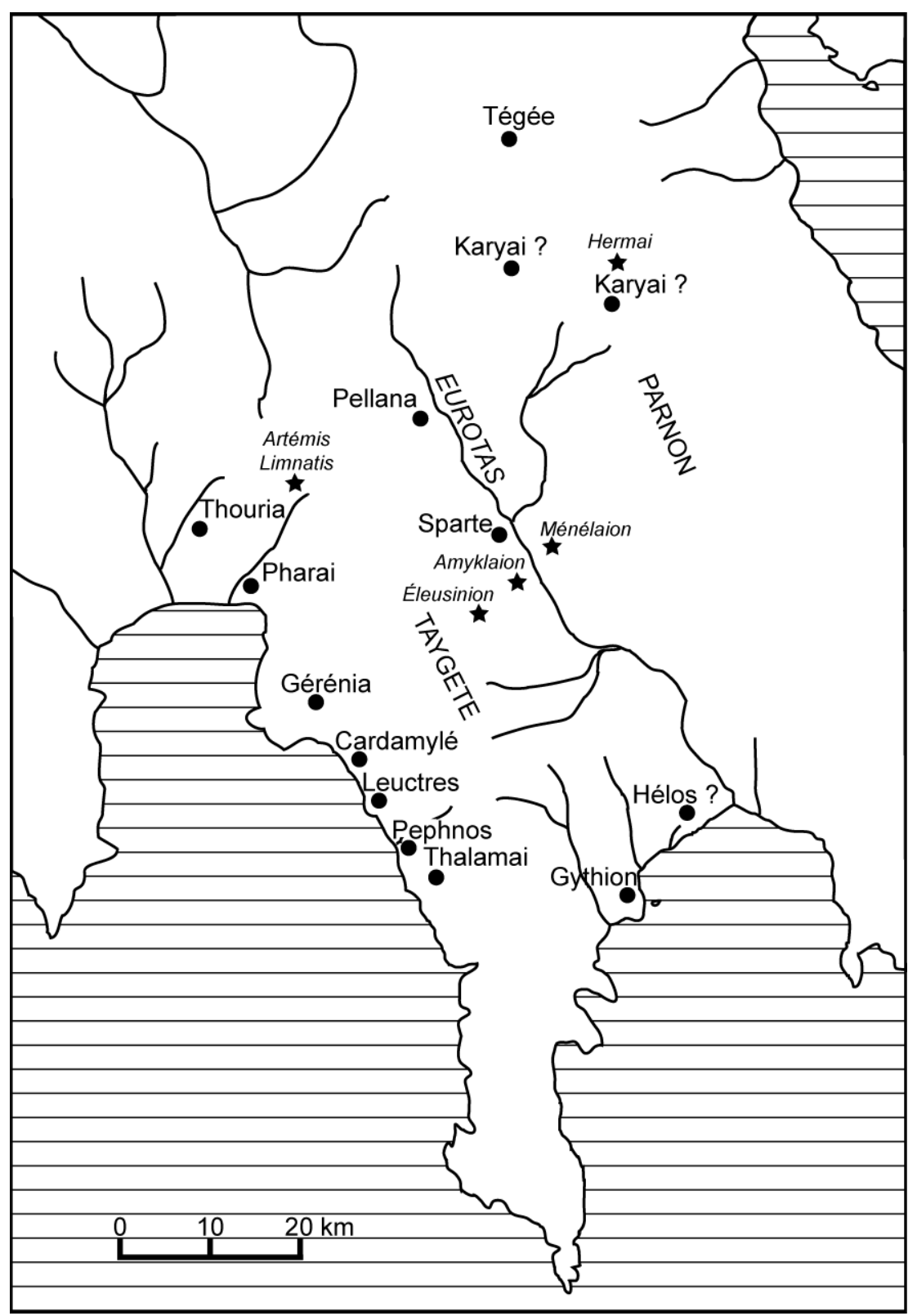

Dans le voisinage du Platanistas, lieu où se tenaient des combats entre éphèbes et dont la description est intégrée à celle du Dromos, immédiatement après la mention de l'hérôon d'Alcon, se trouvaient encore quatre hérôa de 
fils d'Hippocoon : Alcimos, Enaraiphoros, Dorceus et Sébros ${ }^{2}$. Après avoir mentionné ces monuments et fait un bref commentaire sur la poésie d'Alcman, dont la sépulture se trouvait près de l'hérôon de Sébros, Pausanias enchaîne $^{10}$ :

Il y a des sanctuaires d'Hélène et d'Héraclès, de la première près du tombeau d'Alcman, du second tout près de la muraille, et dans ce dernier il y a une statue d'Héraclès en armes : l'aspect de la statue est dû, à ce qu'ils racontent, au combat contre Hippocoon et ses enfants.

Voilà donc, après la mention des monuments héroïques élevés en mémoire des fils d'Hippocoon, que revient une allusion au combat qui les opposa à Héraclès et dont Pausanias va immédiatement après rappeler les origines et le déroulement. Il est assez remarquable de voir comment, jusqu'à ce point du texte, Pausanias a construit son discours autour de ce combat dont le cadre général a été défini dans les deux premiers extraits que nous avons lus. Du trophée dans le Parnon à la statue d'Héraclès en armes au Platanistas, cela fait huit monuments liés à ce combat qui peuvent se lire comme autant de moments de son déroulement, mais à rebours : le trophée, les tombes des vaincus, le vainqueur en armes. C'est précisément à ce point du texte, et à propos de la statue d'Héraclès en armes, que Pausanias choisit de raconter comment tout a commencé ${ }^{11}$.

L'hostilité d'Héraclès, affirment-ils, envers la maison d'Hippocoon, a commencé parce qu'après la mort d'Iphitos, alors qu'il était venu à Sparte pour une purification, ils refusèrent de le purifier. Au commencement de la guerre, survint aussi cette autre raison. Oiônos, un tout jeune homme, mais cousin d'Héraclès - il était en effet un fils de Licymnios, le frère d'Alcmène - vint à Sparte avec Héraclès. Faisant le tour de la cité pour observer, quand il arriva le long de la maison d'Hippocoon, un chien de garde l'attaqua. Mais lui, Oiônos, il se trouve qu'il lance une pierre et abat le chien. Sortent alors en courant les enfants d'Hippocoon et, à coup de bâtons, achèvent Oiônos. Cet événement surtout rendit Héraclès furieux contre Hippocoon et ses enfants. Aussitôt, comme il était en colère, il alla les trouver pour un combat. Il fut alors blessé et se retira sans être vu. Plus tard, après avoir lancé une campagne contre Sparte, il lui fut possible de tirer vengeance d'Hippocoon, mais il tira aussi vengeance de ses fils pour le meurtre d'Oiônos. Et le monument funéraire d'Oiônos a été bâti près de l'Hérakleion.

Il est également remarquable que, après avoir relaté les origines du combat d'Héraclès contre Hippocoon et ses fils, Pausanias embraye à nouveau sur sa description par la mention du tombeau d'Oiônos qui monumentalise précisément le point d'origine de toute l'affaire. Pausanias indique en effet qu'il y avait deux raisons pour lesquelles Héraclès s'en était pris à Hippocoon et à ses fils : le premier a refusé de le purifier, les seconds ont tué Oiônos. Et,

\footnotetext{
9 Paus., III, 15, 1-2. Sur les combats d'éphèbes au Platanistas, voir encore Paus., III, 11, 2 et Lucien, Anacharsis, 38

10 Paus., III, 15, 3.

11 Paus., III, 15, 3-5
} 


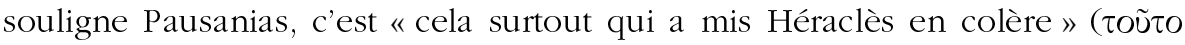

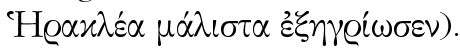

Cette précision justifie en outre l'implication des fils d'Hippocoon dans un épisode qui, tel qu'il avait été présenté auparavant, mettait surtout en jeu la question dynastique. En ce sens, le récit de l'assassinat d'Oiônos et de la colère d'Héraclès apporte a posteriori l'explication de la mention des hérôa des fils d'Hippocoon dans la partie du texte qui précède immédiatement. On voit donc se dessiner une certaine cohérence dans l'espace spartiate décrit par Pausanias. Mais comment faut-il l'interpréter?

Il est bien clair pour nous, comme cela a déjà été maintes fois souligné, que Pausanias construit son discours sur la Grèce, certes autour d'une description topographique, mais pas mécaniquement, ni en fonction d'un cadre infrangible que lui imposerait l'objet de sa description ${ }^{12}$. En d'autres termes, il n'est pas servilement contraint par son sujet à livrer des éléments comme ils se présentent, mais il sélectionne, il met en ouvre, il organise ${ }^{13}$. À plusieurs reprises, dans la description de Sparte, il lui arrive de mentionner un même monument ou un même lieu en deux points différents de son texte et de l'insérer chaque fois dans des réseaux de relation de proximité avec d'autres lieux, d'autres monuments. Ainsi le bâtiment que l'on appelait les Boônèta, est situé près de l'agora, au début de la rue Aphétaïs, en III, 12, 1. Il sert ensuite de point de repère pour localiser le sanctuaire d'Asclépios le plus remarquable de Sparte en III, 15, 10. Autant dire que le sanctuaire d'Asclépios aurait tout aussi bien pu être signalé aux alentours de l'agora ou de l'Aphétaïs. Cette caractéristique de la description topographique mise en ouvre par Pausanias s'explique d'ailleurs sans peine : le texte, à deux dimensions, ne peut présenter que des relations linéaires là où, dans l'espace, les objets dessinent des réseaux de relation simultanément dans toutes les directions. Pausanias choisit donc quelle relation spatiale privilégier, et par conséquent la manière et le moment de parler de tel ou tel sujet.

L'imbrication de la narration dans la description participe donc du travail d'élaboration de Pausanias. Comme l'écrivait Christian Jacob :

La description d'un territoire limité peut se transformer en récit : la promenade de Pausanias relie entre eux différents jalons qui sont autant d'épisodes du mythe; l'ordre du parcours est dès lors déterminant, car il peut seul donner sens au récit ${ }^{14}$.

C'est précisément de cette manière que Pausanias rapporte l'histoire du combat d'Héraclès contre Hippocoon et ses fils. Après un premier mouve-

\footnotetext{
12 Voir notamment C. CALAME, «Représentation discursive du 'panthéon' de Trézène chez Pausanias ", in V. Pirenne-Delforge, Les panthéons des cités des origines à la Périégèse de Pausanias, Liège, 1998 (Kernos, Suppl. 8), p. 149-163.

13 Sur la sélectivité nécessaire de la description, voir J.-M. ADAM, La description, Paris, 1993 (Que sais-je?, 2783), et part. p. 5-25, à propos du rejet presque unanime de la description par les rhétoriciens du XVIII ${ }^{e}$ au $\mathrm{XX}^{\mathrm{e}} \mathrm{s}$. qui n'est pas sans rappeler les critiques adressées à Pausanias à la même époque. Cf. C. Haвicht, Pausanias' Description of Greece, Berkeley, $1998^{2}$ [1985], p. 165175 .

C. JACOB, «Récit de voyage et description », Lalies 1 (1980), p. 131-141, à la p. 133.
} 
ment, implicite, qui conduisait, à travers la succession des bérôa des fils d'Hippocoon, jusqu'au récit des origines de leur différend fatal avec Héraclès, Pausanias va associer explicitement une série de sanctuaires à ces événements.

À l'est du Dromos se trouvait un sanctuaire d'Athéna Axiopoinos dont Pausanias explique l'épiclèse en rapport avec la vengeance («que les hommes de

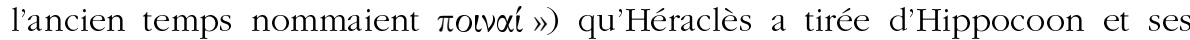
enfants en proportion $\left(\varkappa \alpha \tau^{\prime} \alpha^{\prime} \xi \alpha \nu\right)$ de leurs méfaits ${ }^{15}$. Héraclès a également consacré un sanctuaire d'Héra Aigophagos, "Mange chèvre », pour remercier la déesse de ne pas s'être opposée à lui lors de son combat contre Hippocoon et ses fils. Il aurait, faute d'autre victime, sacrifié des chèvres à la déesse, ce qui expliquerait son épiclèse ${ }^{16}$. Au début de la route de Thérapné se trouvait un temple d'Asclépios Kotyleus consacré par Héraclès. L'épiclèse du dieu viendrait du fait qu'il a guéri Héraclès de sa blessure à la hanche ${ }^{17}$. Et c'est dans le sanctuaire de l'Éleusinion, sur les contreforts du Taygète, qu'Asclépios l'aurait soigné ${ }^{18}$.

Les trois premiers monuments ont chacun une origine liée au combat d'Héraclès. À chaque fois, Pausanias s'appuie sur le récit qu'il a donné en III, 15,3 et y rattache l'aition lié au sanctuaire : le motif de la vengeance pour Athéna Axiopoinos, le succès final pour Héra Aigophagos, la blessure pour Asclépios Kotyleus. À propos de ce dernier, il apporte même des détails complémentaires au récit précédent, à savoir la localisation de la blessure d'Héraclès à la hanche et l'intervention déterminante d'Asclépios. Ce dernier point connaît en outre lui-même un développement lorsque Pausanias précise ensuite que c'est à l'Éleusinion du Taygète qu'Héraclès a été soigné.

La lecture du texte de Pausanias permet donc de reconstruire le déroulement, dans le temps et dans l'espace, de la lutte d'Héraclès contre Hippocoon et ses fils : Héraclès vient à Sparte pour se faire purifier, Hippocoon refuse; Oiônos tue le chien du palais et se fait lyncher par les fils d'Hippocoon; irrité, Héraclès les attaque et se fait blesser; il se retire dans le Taygète où Asclépios le soigne; il revient une fois guéri et tue Hippocoon et ses fils; il consacre des sanctuaires et un trophée; Oiônos et les fils d'Hippocoon reçoivent des honneurs posthumes.

Un élément doit être encore ajouté. Dans sa description de Tégée, Pausanias signale une statue d'Héraclès ${ }^{19}$ :

On a représenté sur sa cuisse une blessure provenant du premier combat qu'il engagea contre les fils d'Hippocoon.

Bien qu'il associe la blessure que porte la statue au combat d'Héraclès, Pausanias n'explique pas la présence de cette statue à Tégée. Il faut se

\footnotetext{
15 Paus., III, 15, 6.

16 Paus., III, 15, 9.

17 Paus., III, 19, 7.

18 Paus., III, 20, 5.

19 Paus., VIII, 53, 9
} 
reporter à Diodore de Sicile ou à Apollodore pour comprendre ${ }^{20}$. D'après ces auteurs en effet, Héraclès s'était assuré l'aide de Cépheus et de ses fils avant de s'attaquer à nouveau à Hippocoon et aux siens. Ces alliés tégéates devaient d'ailleurs payer leur collaboration de leur vie ${ }^{21}$. Pausanias ne développe pas cet épisode ${ }^{22}$, qu'il évoque pourtant brièvement comme une campagne qu'Héraclès aurait lancée pour tirer vengeance d'Hippocoon et de ses fils ${ }^{23}$. Le trophée qui se trouvait près de Karyai pourrait également trouver sa justification dans cet épisode que ne relate pas Pausanias. Ceci révèle cependant que pour le Périégète, le combat contre Hippocoon et ses fils est une affaire exclusivement spartiate.

Précisément, à Sparte, les liens sont très forts entre les espaces jalonnés des souvenirs de la lutte d'Héraclès contre Hippocoon et ses fils. Les activités des éphèbes spartiates assurent la continuité entre le Dromos, le Platanistas et le temple d'Asclépios Kotyleus qui se trouvait sur la route de Thérapné et du Phoibaion $^{24}$. C'est sur le Dromos que les jeunes gens s'entraînaient à la course à l'époque de Pausanias ${ }^{25}$. Les Sphaireis, qui sont les jeunes hommes qui sortent de l'éphébie à Sparte, précise Pausanias, offraient un sacrifice à la statue d'Héraclès qui jouxtait le Dromos, près du monument d'Eumèdès ${ }^{26}$.

Au Platanistas étaient organisés des combats d'éphèbes. Cet endroit était de forme circulaire et entouré d'eau. On y accédait par deux ponts portant, l'un une statue de Lycurgue, l'autre une statue d'Héraclès. Avant le combat, les éphèbes, répartis en deux groupes offraient un sacrifice au Phoibaion dont la victime était un chien. Les échos entre les pratiques des jeunes Spartiates et le récit de la lutte contre les Hippocoontides sont patents. Il faut d'ailleurs souligner que, s'il ne relève pas ces échos, Pausanias choisit néanmoins d'intégrer la mention du rituel du Phoibaion à sa description du Platanistas, peu avant de raconter le combat d'Héraclès, et non dans sa description du

20 Diodore, IV, 33, 5-6; Apollodore, II, 7, 3-4 (143-145).

21 La description de la statue de Tégée assure également l'interprétation de l'origine du nom d'Asclépios Kotyleus. La kotylè en effet, que nous avons identifiée à la hanche, pourrait également désigner la paume de la main. Or, précisément, on lit chez Clément d'Alexandrie, Protreptique II,

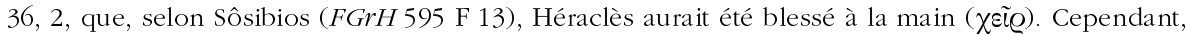
le vocabulaire utilisé par Pausanias correspond exactement à ce que l'on lit chez Homère (Iliade

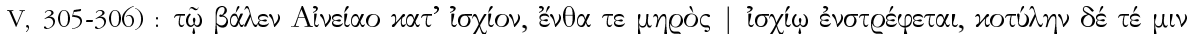
$\varkappa x \lambda \varepsilon ́ o v \sigma$. On peut supposer que Sôsibios situait la blessure d'Héraclès à la kotylè (sans doute à propos de l'Asclépios Kotyleus) et que Clément, ou une source intermédiaire, aura mal interprété le terme.

${ }^{22}$ Il évoque par ailleurs la venue d'Héraclès à Tégée et son union avec Augé, la fille de Cépheus (VIII, 4, 8), événement associé à la guerre contre Hippocoon chez Diodore, IV, 33 et Apollodore, II, 7, 3-4 (143-145). Pausanias évoque aussi le cheveu de Méduse qui protégeait Tégée de toute agression, mais il en attribue le don à Athéna, là où Apollodore rapporte que c'est Héraclès qui l'a donné à Cépheus pour le convaincre de l'accompagner à Sparte sans craindre que Tégée fut prise en son absence.

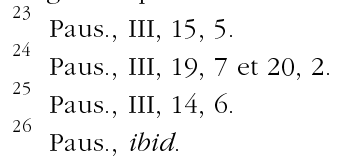


Phoibaion. Mais ce parallélisme, que l'on pourrait développer, est-il une survivance, une réalité ancienne, dont Pausanias aurait été le témoin privilégié ? Pas nécessairement et, en tout cas, pas uniquement, car il semble s'inscrire dans le cadre de pratiques bien vivantes à l'époque de Pausanias.

Comme l'a rappelé Yves Lafond ${ }^{27}$, les cités péloponnésiennes comme Sparte ou Argos connaissaient, à l'époque impériale, une forte activité de rénovation de leurs institutions éducatives et athlétiques. À Sparte, les principaux magistrats avaient pour charge d'encadrer entraînement et concours pour les jeunes gens au théâtre, dans les gymnases du Dromos et sur le Platanistas $^{28}$. À cette époque (du I $\mathrm{I}^{\mathrm{er}}$ au début du $\mathrm{III}^{\mathrm{e}}$ siècle ap. J.-C.), quelques grandes familles cumulaient les actes d'évergétisme, les charges de magistrats et les prêtrises. L'une d'entre elles, d'ancienne citoyenneté romaine, apparaît de manière récurrente dans les documents d'époque antonine : les Tiberii Claudii.

Une inscription honorifique de Sparte porte le texte suivant ${ }^{29}$ :

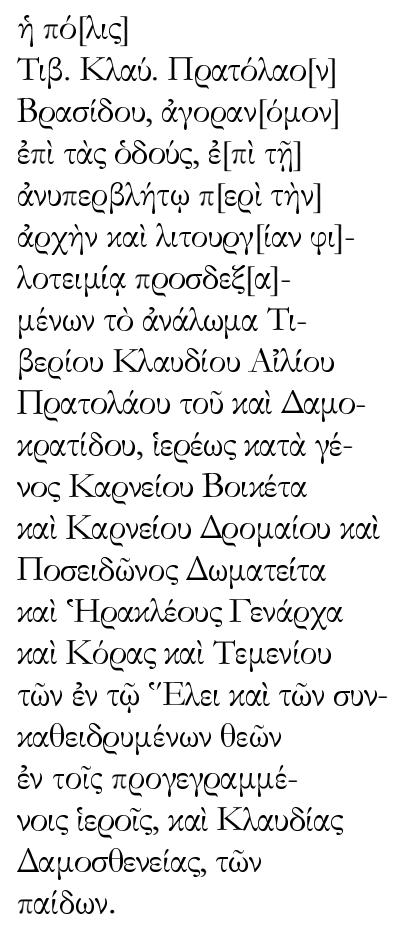

Les Tiberii Claudii constituaient une des plus grandes familles de Sparte qui avait des ramifications dans l'ensemble de la province d'Achaïe. Elle était

\footnotetext{
${ }^{27}$ Voir sa contribution ici-même, avec P. MARCHETTI, Le nymphée de l'agora d'Argos : fouille, étude architecturale et historique, Athènes, 1995 (Études Péloponnésiennes, 11), 1995, p. 191-201.

${ }^{29} I G \mathrm{~V}$ 1, 497. Pour le commentaire de cette inscription, voir A. HupfloHER, Kulte im kaiserzeitlichen Sparta. Eine Rekonstruktion anhand der Priesterämter, Berlin, 2000, p. 125-138.
} 
notamment liée avec la famille d'Hérode Atticus, qui avait lui-même reçu l'éducation spartiate ${ }^{30}$. Tiberios Claudios Brasidas fut sénateur sous Marc Aurèle, comme nous l'apprend un passage du Codex Iustinianus ${ }^{31}$. Spartiatikos, un des fils de Brasidas, et Eudamos le fils de Spartiatikos ont été grandsprêtres du culte impérial à Sparte ${ }^{32}$. La prééminence des Tiberii Claudii à Sparte explique le nombre des cultes dont ils assumaient la prêtrise, charge sans doute coûteuse mais de grand prestige ${ }^{33}$.

Trois générations de Tiberii Claudii sont représentées ici : Brasidas, Pratolaos l'ancien, Pratolaos le jeune qui est aussi appelé Damocratidas et sa soeur Damosthéneia. Le second Pratolaos, qui porte un double gentilice, doit être un fils adoptif ${ }^{34}$. Il semble toutefois que la prêtrise qu'il assumait de manière

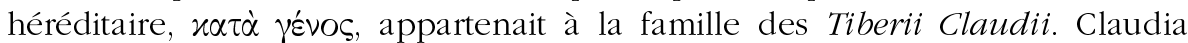
Damosthéneia l'assumera également ${ }^{35}$, soit qu'elle l'ait hérité de son frère, soit que la prêtrise ait été assumée conjointement par un homme et une femme de la famille et que Claudia Damosthéneia l'ait prise en charge à la suite de la collègue supposée de Damocratidas à une époque postérieure à cette inscription, que l'on peut dater du début du III $^{e}$ siècle ap. J.-C.

Les cultes mentionnés dans l'inscription sont pour la plupart connus de Pausanias. Carneios Oiketas « recevait des honneurs à Sparte même avant que reviennent les Héraclides ${ }^{36}$. Carneios Dromaios peut être identifié au second sanctuaire spartiate de Carneios signalé par Pausanias en dehors du Dromos, du côté de la statue d'Héraclès à laquelle sacrifiaient les Sphaireis dont la mention était associée à celle du monument funéraire d'Eumédès ${ }^{37}$. Quant à Poseidon Dômatitès, il se trouvait près de l'hérôon d'Alcon que Pausanias signale juste avant d'aborder la description du Platanistas ${ }^{38}$. Cette contiguité dans le texte n'implique nullement une proximité topographique ${ }^{39}$. Du moins,

30 Outre l'étude de A. SPAWFORTH, «Families at Roman Sparta and Epidaurus: Some Prosopographical Notes », ABSA 80 (1985), p. 191-258, aux p. 224-241, voir les remarques de MARCHETTI, o.c. (n. 27), p. 197-199.

31 XXXVI, 1, 23. Le passage évoque un jugement dans une affaire d'héritage qui doit avoir été rendu en 173 ou 174 lorsque, depuis le théâtre de la guerre contre les Sarmates, à Carnutum ou à Sirmium, Marc Aurèle traita les affaires de Grèce : A. BIRLEy, Marcus Aurelius, a Biography, Londres, $2000^{3}$ [1966], p. 180.

32 Hupfloher, o.c. (n. 29), p. 150 avec les références. Le nom de Spartiatikos porté par un fils de Brasidas fait supposer à A. Spawforth un lien des Tiberii Claudii avec la grande famille spartiate des Euryclès.

33 CARTLEDGE - SPAWFORTH, o.c. (n. 4), p. 164-165 et 184-189. Sur les charges de prêtres dans la Sparte impériale, voir A. SPAWFORTH, «Spartan Cults under the Roman Empire: Some Notes », in J.M. SANDERS (éd.), Philolakôn. Lakonian Studies in Honour of Hector Catling, Londres, 1992, p. 230-233 et maintenant HuPFLOHER, o.c. (n. 32)

34 SPAWFORTH, l.c. (n. 30), p. 232-233.

35 Cf. IG V 1, 608.

36 Paus., III, 13, 3

37 Paus., III, 14, 6

38 Paus., III, 14, 7

39 D'autant plus que, même lorsque Pausanias situe les éléments de sa description les uns par rapport aux autres, la proximité réelle de ces éléments est difficile à évaluer, tant les rapports 
rien, en l'état actuel des prospections archéologiques, ne nous permet de le vérifier. En revanche, il est très significatif que Pausanias ait choisi de signaler précisément ces monuments, et de les associer dans son texte. Et il apparaît ainsi que les lieux associés par Pausanias à la lutte d'Héraclès contre Hippocoon et ses fils sont parsemés de sanctuaires dont les prêtrises étaient, au début du III $^{e}$ siècle et sans doute avant, détenues par la famille des Tiberii Claudii.

D’après Pausanias, un xoanon de Corè était régulièrement emmené de la localité côtière d'Hélos à l'Éleusinion du Taygète, sans doute en procession ${ }^{40}$. Il n'est pas certain que l'Hélos de l'inscription doive être identifiée à l'antique cité du rivage laconien qui était en ruine à l'époque de Pausanias ${ }^{41}$. En effet, Patrick Marchetti ${ }^{42}$ a attiré l'attention sur le fait qu'il devait plutôt s'agir d'un quartier de la ville de Sparte, un ancien marais, rejoignant ainsi l'opinion des premiers éditeurs des inscriptions spartiates, Boeckh et $\mathrm{Tod}^{43}$. Certes, le fait que l'Hélos de la côte ait été en ruine au II 'e siècle ap. J.-C. n'empêche nullement qu'on y ait entretenu un culte ${ }^{44}$. Cela ne semble d'ailleurs nullement étonner Pausanias. Cependant, un sanctuaire situé également év $\tau \tilde{\omega}{ }^{~}{ }^{\prime} E \lambda \varepsilon \iota$ dans une autre inscription semble être identique à un sanctuaire urbain signalé par Pausanias $^{45}$, ce qui invite à distinguer le quartier de Sparte et la cité «homérique ».

Quoi qu'il en soit, l'important pour nous est le lien entre l'Éleusinion et la Corè de l'Hélos, que Pausanias ait confondu la cité et le quartier du même nom ou pas ${ }^{46}$. Car ce lien est renforcé par le fait que la prêtrise, héréditaire, de Démétèr Eleusinia appartenait également à la famille des Tiberii Claudii et

établis sont lâches. Ainsi, l'hérôon d'Alcon se rencontre «en avançant un peu " (ỏnígov

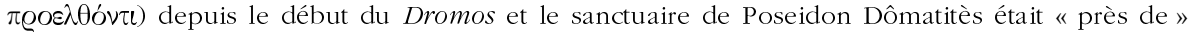
$(\pi \alpha \varrho \alpha ́)$ l'hérôon d'Alcon.

40 Paus., III, 20, 7. Voir la carte ci-dessus.

41 Paus., III, 22, 3. Hélos semble être un village ( $\omega^{\prime} \mu \eta$ ) à l'époque de Strabon, qui rappelle que c'était autrefois une cité, au témoignage d'Homère (Strabon, VIII, 5, 2 [C363] citant Iliade II, 584). Cf. D.G.J. SHIPLEY, "'The Other Lakedaimonians': The Dependent Perioikic Poleis of Laconia and Messenia ", in M.H. HANSEN (éd.), The polis as an urban centre and as a political community, Copenhagen, 1997 (Historisk-filosofiske Meddelelser / Det Kongelige Danske Videnskabernes Selskab, 75. Acts of the Copenhagen Polis Centre, 4), p. 189-281, aux p. 252-253, $\mathrm{n}^{\circ} 65$ avec la bibliographie.

42 MARchetTi, Le nymphée, o.c. (n. 27), p. 211-216 et « Le 'Dromos'», l.c. (n. 7), p. 157.

43 Voir le commentaire de Boeckh à $C I G, 1444$ (t. II, p. 683) et celui de Tod dans M.N. TOD \& A.J.B. WACE, A Catalogue of the Sparta Museum, Oxford, 1906, p. 81, n 691.

44 Sur la question de l'abandon ou de la persistance des lieux de cultes dans les habitats désertés à l'époque romaine, voir S.E. Alcock, "Minding the Gap in Hellenistic and Roman Greece ", in ead. \& R. OsBonne, Placing the Gods, Oxford, 1994, p. 247-261 et ead., Graecia Capta. The landscapes of Roman Greece, Cambridge, 1992, p. 207-210.

45 Cf. Asclépios Schoinatas d'IG V 1, 602 et Asclépios Agnitas de Paus., III, 14, 7.

46 A. Spawforth, suivi par S.E. Alcock, a supposé que les grandes familles de Sparte possédaient des terres dans la plaine maritime d'Hélos. La procession attesterait la domination sur ce territoire dans le cadre de rivalités de prestige entre familles de l'élite, voire entre cités: CARTLEdge - SPAWforTh, o.c. (n. 4), p. 137-138; AlCOCK, Graecia Capta, o.c. (n. 44), p. 203-205 et 212. 
notamment à une petite-fille présumée de Brasidas, Claudia Agéta ${ }^{47}$. C'est d'ailleurs à l'Éleusinion qu'a été retrouvée la base de statue portant l'inscription honorifique pour Claudia Damosthéneia, elle aussi «prêtresse à vie et héréditaire de Carneios Oiketas, Carneios Dromaios, Poseidon Domatitès, Héraclès Génarchas, Kora et Téménios dans l'Hélos, les dieux conjoints à ceux-ci et (précise ce texte) les autres dieux " ${ }^{48}$. C'est à l'Éleusinion rappelonsle que, selon Pausanias, Héraclès avait été caché par Asclépios pour soigner sa blessure. Que l'Hélos de l'inscription soit l'agglomération côtière ou un quartier de Sparte, comme nous inclinerions à le penser, n'enlève rien au lien qui associe le culte de Corè avec l'Éleusinion et avec les Tiberii Claudii.

La mise en parallèle de l'inscription de Pratolaos avec la description de Pausanias révèle donc la cohérence des charges religieuses assurées par les Tiberii Claudii. Elle révèle également que le discours de Pausanias rejoint, de manière très significative, les réalités cultuelles de son temps.

Dans le récit du combat contre Hippocoon et ses fils, dans l'espace de Sparte que décrit Pausanias et dans les prêtrises assumées par les Tiberii Claudii, Héraclès est omniprésent. Pratolaos puis Damosthéneia furent prêtres d'Héraclès Génarchas. Plusieurs familles de Sparte à l'époque impériale se réclamaient encore d'une ascendance héraclide, comme les familles royales de jadis $^{49}$. Or, nous l'avons vu, la victoire d'Héraclès sur Hippocoon était au coeur de la prétention des Héraclides au trône de Sparte. Prétentions familiales, limitées à quelques membres de l'élite ou prétentions collectives relayées par cette même élite ? La revendication d'une origine Héraclide semble en tout cas encore bien présente à Sparte au II siècle ap. J.-C.

\section{Les Héraclides}

En 25 ap. J.-C., un différend entre Messéniens et Lacédémoniens est porté devant le sénat. Il concerne le sanctuaire d'Artémis Limnatis :

Auditae debinc Lacedaemoniorum et Messeniorum legationes de iure templi Dianae Limnatidis, quod suis a maioribus suaque in terra dicatum Lacedaemonii firmabant (...). Contra Messenii ueterem inter Herculis posteros diuisionem Peloponnesi protulere, suoque regi Denthaliatem agrum in quo id delubrum cessisse (...).

On entendit ensuite les délégués de Lacédémone et de Messène sur le statut juridique du temple de Diane Limnatis. C'était par leurs ancêtres et sur leur territoire qu'il avait été consacré, affirmaient les Lacédémoniens (...). De leur côté, les Messéniens alléguèrent un ancien partage du Péloponnèse entre les descendants

\footnotetext{
${ }^{47}$ IG V 1, 249, avec SPAWFORTH, l.c. (n. 30), p. 230-231 et S. WALKER, "Two Spartan Women and the Eleusinion ", in ead. \& A. CAMERON (éds), The Greek renaissance in the Roman empire, Londres, 1989 (BICS, Suppl. 55), p. 130-141.

${ }^{48} I G \mathrm{~V} \mathrm{1}, 608, c f$. également $I G \mathrm{~V} 1,589$ qui est probablement un second exemplaire du même texte.

49 Voir encore la contribution d'Yves Lafond ici-même.
} 
d'Hercule : c'est à leur roi qu'était échu le territoire de Denthalia où se trouvait le temple $(\ldots)^{50}$.

Un sanctuaire de ce nom avait été, selon Pausanias notamment, le théâtre des événements qui devaient provoquer la première guerre de Messénie ${ }^{51}$. Les Lacédémoniens affirmaient que des Messéniens avaient violenté des jeunes Lacédémoniennes qui assistaient à la fête de la déesse et tué le roi Téléclos qui avait voulu les défendre. Les jeunes filles honteuses se seraient pendues. Les Messéniens pour leur part prétendaient que c'était une embuscade, que des jeunes gens imberbes s'étaient déguisés en jeunes filles pour attaquer les plus éminents des Messéniens qui, en se défendant, avaient tué les jeunes gens et le roi Téléclos qui les menait.

Voilà ce que les uns et les autres disent, que tout un chacun croie ce que lui inspire l'intérêt qu'il porte aux uns ou aux autres ${ }^{52}$.

Je ne reviendrai pas ici sur l'interprétation de ces récits qu'ont très bien analysés Claude Calame et Pierre Ellinger ${ }^{53}$. Je relèverai seulement l'insistance de Pausanias à souligner le différend et son association au partage du Péloponnèse, car ceci montre l'actualité de la tradition du retour des Héraclides en pleine époque impériale.

La référence aux Héraclides, qui apparaît dans le texte de Tacite à propos des revendications messéniennes sur le sanctuaire d'Artémis Limnatis, n'est pas isolée, bien au contraire. Il est même possible d'identifier un discours persistant qui, de la fin de l'époque classique à l'époque romaine, construit et entretient l'image des frontières des cités doriennes du Péloponnèse comme un reflet de l'antique partage des Héraclides.

Au lendemain de la refondation de Messène par Épaminondas, après la défaite de Sparte à Leuctres en 371 av. J.-C., Isocrate prêtait à Archidamos, prince héritier de Sparte et Héraclide, une argumentation s'appuyant sur la tradition du retour des Héraclides pour contester leurs droits aux Messéniens. Trente ans après, en 337, la ligue de Corinthe mise en place par Philippe II de Macédoine au lendemain de son succès de Chéronée fixait les frontières entre Sparte et les États voisins sur la base d'une reconstruction érudite du partage

50 Tacite, Annales IV, 43, 1-2. Texte et trad. repris à Tacite, Annales, t. II : Livres IV-VI, texte établi et traduit par P. Wuilleumier, deuxième tirage revu et corrigé par H. LE BONNIEC, Paris, 1990 (Collection des Universités de France).

51 Paus., IV, 4, 2.

52 Paus., IV, 4, 2. Cf. III, 2, 6 et III, 7, 4.

53 C. CALAME, Les Chours de jeunes filles en Grèce archä̈que, t. I : Morphologie, fonction religieuse et sociale, Rome, 1977 (Filologia e critica, 20), p. 253-264, 2 éd. anglaise : Choruses of Young Women in Ancient Greece. Their Morphology, Religious Role, and Social Functions, Lanham, 1997, p. 142-149 et id., "Discours mythique et discours historique dans trois textes de Pausanias », Degrés 17 (1979), p. 1-30. P. Ellinger, La légende nationale phocidienne. Artémis, les situations extrêmes et les récits de guerre d'anéantissement, Athènes, 1993 (BCH, Suppl. 27), p. 4143. 
du Péloponnèse entre les Héraclides, qui pourrait remonter à l'école d'Aristote ${ }^{54}$.

D'après un arbitrage rendu par la Ligue achéenne à propos d'un différend de frontière entre Sparte et Mégalopolis, une décision des vainqueurs de Sellasie, en 222, avait également veillé à maintenir Sparte dans les frontières du partage des Héraclides ${ }^{55}$. C'était encore, nous l'avons vu, l'argument héraclide que mettaient en avant les Messéniens devant Tibère.

Après 42, le territoire en litige avec les Messéniens avait été rendu aux Spartiates par Octave et Marc Antoine ${ }^{56}$. Il avait ensuite été restitué à Messène, décision confirmée par Tibère en 27 ap. J.-C. Près d'un siècle et demi plus tard, les problèmes de frontières entre Sparte et Messène trouvent encore un écho chez Pausanias, comme nous l'avons vu, dans l'exposé généalogique qui ouvre ses Lakônika et comme nous allons le voir dans l'examen des lieux d'exil de Tyndare, second volet de sa présentation de l'histoire d'Hippocoon et ses fils.

\section{Tyndare et Hippocoon}

À la centralité des monuments liés au combat d'Héraclès s'oppose logiquement l'éloignement des lieux d'exils de Tyndare. Selon Pausanias, ce serait Thalamai ou Pellana qui passaient pour l'avoir accueilli. ${ }^{57}$. C'est sur l'îlot de Pephnos que seraient nés les Dioscures, précise Pausanias, et Hermès les auraient ensuite emmenés à Pellana. Cette dernière précision permet, en quelque sorte, de réconcilier les deux versions de l'exil de Tyndare, la messénienne et la lacédémonienne, mais Pausanias ne le relève pas.

Thalamai et Pephnos sont les premiers établissements sur le versant sud du Taygète pour lesquels Pausanias fait état de revendications messéniennes ${ }^{58}$. Les Messéniens prétendaient en effet que Pephnos, jadis, leur appartenait et que les Dioscures qui y étaient nés étaient également Messéniens. Pausanias ne se prononce pas ici sur ces revendications, mais il a dit ailleurs son opinion sur le passé messénien. Les Spartiates en effet prétendaient avoir dans leur cité les sépultures d'Idas et Lyncée, les fils d'Apharée, mort sous les coups des Dioscures et de Zeus. Pour Pausanias, il est plus vraisemblable que les fils d'Apharée avaient été ensevelis en Messénie :

Mais les malheurs des Messéniens et le temps durant lequel ils se sont exilés du Péloponnèse, ont jeté dans l'oubli nombre de traditions anciennes même pour

54 M. PIÉRART, «Argos, Philippe II et la Cynourie (Thyréatide) : les frontières du partage des Héraclides », in R. FREI-STOLBA \& K. GEX (éds), Recherches récentes sur le monde hellénistique, Berne, 2001 (Echo, 1), p. 27-43.

55 Iv $0,47=$ Syll. ${ }^{3}, 665$.

56 Tacite, Annales IV, 43, 1.

57 Comme le précise Pausanias en III, 1, 4. Ce dernier ne manque d'ailleurs pas de le rappeler quand son cheminement descriptif l'amène à évoquer ces localités. $C f$. Paus., III, 21, 2 pour Pellana et 26, 2-3 pour Thalamai, où il n'évoque pas directement l'exil de Tyndare, mais bien la naissance des Dioscures qu'il date de cet exil en III, 1, 4.

58 Voir la carte ci-dessus. 
ceux qui sont revenus, et puisqu'ils ne savent pas, il est désormais possible, à ceux qui le veulent, de les leur disputer ${ }^{59}$.

Pausanias inclinerait-il dès lors à croire les Messéniens et à faire passer le Taygète aux Dioscures? Il revient en tout cas dans sa description de Messène sur ces prétentions, en des termes beaucoup plus amènes que lorsqu'il s'agissait des fils d'Apharée à Sparte ${ }^{60}$ :

Il y a un sanctuaire vénérable de Déméter et des statues des Dioscures enlevant les filles de Leucippe. Et moi, cela aussi je l'ai déjà montré dans ce qui précède, à savoir que les Messéniens revendiquent que les enfants de Tyndare leur appartiennent à eux et non aux Lacédémoniens.

Les Messéniens, en tout cas, revendiquaient Pephnos, et sans doute Thalamai que Pausanias baptise en III, 1, 4 «Thalamai de Messénie ». Ce sont les gens de Thalamai qui affirment que les Dioscures sont nés à Pephnos. Les Messéniens revendiquaient aussi Leuctres, plus au sud, où, une fois encore, les héros et les cultes messéniens et lacédémoniens sont aux prises.

Leuctres tirerait son nom de Leucippe, à ce que disent les Messéniens. Pausanias serait d'ailleurs prêt à le croire, car cela expliquerait la ferveur particulière avec laquelle est honoré Asclépios. Asclépios en effet est un fils d'Arsinoé, la fille de Leucippe, pensent les Messéniens. A Leuctres il y a aussi un xoanon d'Apollon Carneios comme en révèrent d'habitude les Lacédémoniens de Sparte ${ }^{61}$. Et du temps de Pausanias, il est encore arrivé cet événement étrange : alors qu'un incendie avait ravagé une forêt, on y trouva une statue de Zeus Ithômatas, preuve avancée par les Messéniens pour dire que cette région leur appartenait jadis. "Mais il serait possible aussi que, des Lacédémoniens habitant Leuctres depuis le début, Zeus Ithômatas ait eu chez eux un culte », nuance Pausanias ${ }^{62}$. Une étymologie, un culte d'Asclépios, un Zeus Ithômatas pour les Messéniens, Apollon Carneios pour les Lacédémoniens. Pausanias ne semble pas vouloir trancher, mais rapporte encore fidèlement les revendications messéniennes.

En revanche, il ne fait pas de doute pour Pausanias que Cardamylé, au nord de Leuctres, est en Messénie. C'est Auguste, selon lui, qui l'en a retranchée pour la donner aux spartiates. Le Périégète y signale un Apollon Carnéios, honoré selon l'usage local des Doriens. De même, à Gérénia, se sont des Messéniens rattachés aux Laconiens Libres, la ligue de cités, majoritairement d'anciens établissements périèques, organisée ou réorganisée

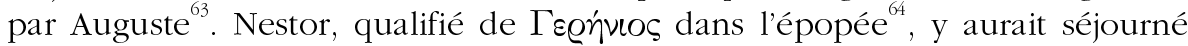

59 Paus., III, 13, 2.

Paus., IV, 31, 9.

Il se trouvait à Leuctres également un temple de Cassandre, appelée alors Alexandra, comme le précise Pausanias, sans établir de lien toutefois avec le culte rendu à cette même Alexandra à Amyclées dont il a fait état en III, 19, 6.

${ }^{62}$ Paus., III, 26, 4-6.

63 Voir CARTLEDGE - SPAWFORTH, o.c. (n. 4), p. 101 et 173-174 
après que Pylos avait été prise par Héraclès. Les extrêmes se rejoignent: Gérénia pour Nestor répond à Thalamai pour Tyndare. Et c'est au nord de Gérénia que, du temps de Pausanias, passait la frontière ${ }^{65}$ :

Les Messéniens, du côté de leur terre cédée par l'empereur au Lakônikon, ont à notre époque pour frontière avec Gérènia le «Gouffre du Porc », comme on le nomme.

Pausanias adopte ici un vocabulaire spécifique, rendu nécessaire par le fait que, à ses yeux, il ne s'agit pas exactement de la frontière entre la Lakonikè et la Messénie.

Pour Pausanias en effet, la Messénie commençait sans doute à Thalamai et certainement à Cardamylè. Et la redistribution de l'espace sous Auguste n'y change rien ${ }^{66}$. Dès lors, il est aisé de comprendre pourquoi Pausanias parle des «Messéniens, du côté de leur terre cédée par l'empereur au Lakônikon », et pas de Messénie : la Messénie commence beaucoup plus au sud. C'est pour la même raison qu'il parle de Lakônikon et pas de Lakônikè. Nous pensons que Pausanias désigne par le terme Lakônikon l'ensemble formé par Sparte et les cités des Laconiens Libres. Cet ensemble qui ne correspond pas exactement avec une région précise, puisqu'il déborde sur la Messénie, n'équivaut pas non plus à un ensemble politique unique. Il est néanmoins « Laconique », puisqu'il réunit Sparte et les Laconiens Libres.

On pourrait voir une confirmation de cette lecture dans une inscription de l'époque de Vespasien (78 ap. J.-C.). Il s'agit d'un inventaire des bornes placées à la frontière entre la Messénie et la Laconie, dressé par un géomètre romain. Il y est question de «la combe qu'ils dénomment Choireion, qui marque la frontière entre Messène et Lacédémone du côté des Laconiens Libres ${ }^{67}$. Le document confirme la frontière signalée par Pausanias en des termes tellement proches que l'on peut d'ailleurs se demander si celui-ci n'en a pas eu connaissance. Mais l'inscription parle de la frontière de Messène avec "Lacédémone du côté des Laconiens Libres », ce qui n'est pas tout à fait la même chose que la frontière avec les Laconiens Libres. A l'époque classique, le nom Lacédémone a pu désigner la cité de Sparte et les cités périèques qui

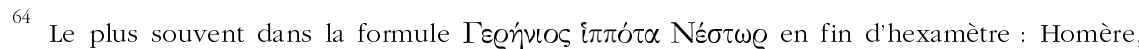

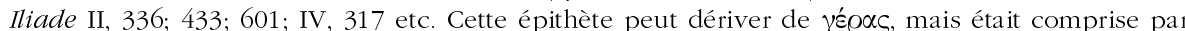
les anciens comme un adjectif dérivé d'un toponyme. Cf. Strabon, VIII, 4, 4 (C352) avec le commentaire de P. Chantraine, Dictionnaire étymologique de la langue grecque, Paris, 1968, s.v. et la communication de F. BADER résumée dans REG 92 (1979), p. X-XI.

${ }^{65}$ Paus., IV, 1, 1.

66 Sur tout ceci, voir aussi Ch. LE ROY, «Pausanias et la Laconie ou la recherche d'un équilibre », in D. KNOEPFLER \& M. PIÉRART (éds), Éditer, traduire, commenter Pausanias en l'an 2000, Genève, 2001 (Université de Neuchâtel. Recueil des travaux publiés par la faculté des lettres et sciences humaines, 49), p. 223-237.

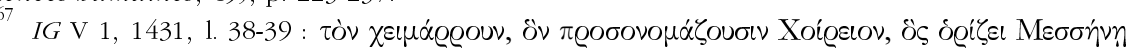

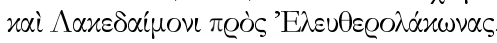


lui étaient soumises, sans référence à la géographie physique ${ }^{68}$. On pourrait dès lors voir un prolongement de ce sens dans l'inscription de Messène, où Lacédémone renverrait à l'ensemble territorial formé par Sparte et la ligue des Laconiens Libres, perpétuant, selon de nouvelles modalités, l'ensemble de jadis ${ }^{69}$.

Quant à Pharai, voisine de Gérénia, elle a été, à l'époque d'Auguste, rattachée sans solution de continuité à l'ensemble dont le «Gouffre du porc » marque la limite avec la Messénie à l'époque de Vespasien, c'est-à-dire le Lakônikon de IV, 1, 1. Cette zone, dans l'esprit de Pausanias, se serait donc étendue plus au nord à l'époque d'Auguste. Comme Jacqueline Christien l'a montré, il était impératif de détacher Pharai de Messène dans la mesure où elle se trouvait sur la route entre Thouria et Sparte ${ }^{70}$. Or Thouria avait été attribuée à Sparte par Auguste également ${ }^{71}$.

Le sanctuaire d'Artémis Limnatis où Pausanias place l'épisode de Téléclos se trouvait précisément, selon lui, dans l'arrière pays de Thouria $^{72}$ :

Il y a à l'intérieur des terres un village, Kalamai, et Limnai, un lieu-dit. Là, il y a un sanctuaire de Limnatis Artémis où, dit-on, Téléclos, qui régnait sur Sparte, trouva la mort.

Lorsque, après Actium, Auguste réorganisa le sud de la Laconie, il enleva Thouria et Cardamylé aux Messéniens et les rattacha à Sparte, peut-être pour compenser la perte de Gythion devenue autonome au sein de la Ligue des Laconiens Libres. Pharai, quant à elle, était rattachée à cette même Ligue, de même que Gérénia ${ }^{73}$. Toutes ces cités se distribuent autour du haut Nédon et de la région de Volimnos. Ce lieu, à la sortie de la passe de Langada, qui

68 Voir l'étude de J. HALL, «Sparta, Lakedaimon and the nature of perioikic dependency », in P. Flensted-JEnSEN, Further Studies in the Ancient Greek Polis, Stuttgart, 2000, p. 73-89, à la p. 81.

69 En tout cas, la zone où se situe les cités des Laconiens Libres est loin d'être homogène. Les cités indépendantes et les établissements dépendants de Sparte y alternent. Il serait donc possible que le «Gouffre du porc » marque effectivement la frontière entre la Messénie et une zone dépendant de Sparte, même si la majorité de cet espace se définit politiquement comme le territoire des cités de la Ligue des Laconiens Libres.

${ }^{70} \mathrm{~J}$. CHRISTIEN, «Les liaisons entre Sparte et son territoire malgré l'encadrement montagneux », in J.-F. BERGIER (éd.), Montagnes, flewves, forêts dans l'bistoire. Barrières ou lignes de convergence? Berge, Flüsse, Wälder in der Geschichte. Hindernisse oder Begegnungsräume? Travaux présentés au XVI congrès international des sciences historiques, Stuttgart, août 1985, St. Katharinen, 1989, p. 18-44, à la p. 31.

71 Paus., IV, 31, 1-4.

72 Paus., IV, 31, 3 .

73 Paus., III, 26, 7-11; IV, 30, 2; IV, 31, 1. Le sort de la cité d'Abia n'est pas clair. Cette cité se trouvait sur la côte du golfe de Messénie, entre le Choireion et Pharai (Paus., IV, 30, 1-2), mais le Périégète ne dit pas si elle a fait partie de la Ligue des Laconiens Libres. Selon nous, c'est probable, car il est difficile d'imaginer qu'Auguste ait laissé cette enclave messénienne au cœur des territoires qu'il souhaitait rattacher à l'ensemble lacédémonien. Quand, en 182 av. J.-C., la Messénie fut à nouveau admise au sein de la Confédération Achéenne, après sa défection de 184 env., trois cités adhérèrent de manière indépendante: Thouria, Pharai et Abia (Polybe, XXIII, 17, 2), c'est-à-dire les trois cités les plus proches de la frontière avec Sparte, qui, précisément, rejoint elle aussi, au même moment, la Confédération (Polybe, XXIII, 17, 5). 
permet encore aujourd'hui de franchir le Taygète, constituait un nœud routier et commandait les relations entre Sparte et la Messénie ${ }^{74}$. Ceci expliquerait à la fois pourquoi les récits sur l'origine de la guerre de Messénie se sont cristallisés sur le sanctuaire d'Artémis Limnatis, mais aussi pourquoi la région qui l'entoure a été si âprement disputée durant toute l'antiquité et jusqu'au II ${ }^{\mathrm{e}}$ siècle ap. J.-C. À l'époque de Trajan, Thouria revendiquait encore Lacédémone comme sa métropole ${ }^{75}$.

Après ces tours et détours, à la suite de Pausanias et un peu au-delà, sur les traces de Tyndare, Héraclès, Hippocoon et ses fils dans la Sparte de la fin du II $^{\mathrm{e}}$ et du début du III $^{\mathrm{e}}$ siècle ap. J.-C., deux enseignements corollaires se dégagent. Tout d'abord, le discours de Pausanias apparaît extrêmement cohérent et construit. Ensuite, il semble rencontrer les préoccupations de son temps.

On ne peut dire à partir des revendications généalogiques des familles de l'élite spartiate ou des complexes cultuels dont elles assumaient la prêtrise, si ces familles ou la société spartiate dans son ensemble de l'époque des Antonins auraient reconnu leurs propres discours dans la Périégèse. Mais le devoir de mémoire que s'est assigné Pausanias semble porter sur les mêmes objets. Et la constatation vaut également pour les Messéniens.

Il semble que Pausanias veuille restituer à ces derniers un passé qu'euxmêmes, à l'en croire, ont perdu. Cependant, ceux-ci ne l'ont manifestement pas attendu, ni les Spartiates pour leur répondre. En effet, tandis qu'un citoyen de Messène était honoré comme nouvel Épaminondas ${ }^{76}$, les Spartiates faisaient bénéficier un des leurs d'une exemption d'impôt du fait que son ancêtre avait tué Épaminondas à la bataille de Mantinée ${ }^{77}$. Jeux et enjeux de mémoire longtemps ressassée, qui engagent en définitive à reconsidérer l'histoire religieuse des cités du Péloponnèse à l'époque romaine et invitent à redoubler de prudence dans l'exploitation du témoignage de Pausanias pour d'autres époques que la sienne.

O. GENGLER

Rue Sous l'Eau, 10

B - 4020 LIÈGE

Courriel : ogengler@yaboo:fr

74 CHRistien, l.c. (n. 70), p. 30-34

IG $\mathrm{V} 1,1381$

${ }_{77}$ Iv $O, 447$ honorant Tiberius Claudius Crispianus.

77 Kallikratès, un contemporain de Plutarque : Agésilas, 35, 1-2. 